\title{
Study on Growth and Phenotypic Characters of Different Clones of Poplar (Populus deltoides Marsh.) in Nursery
}

\author{
Rajiv Kumar ${ }^{1}$, Bimlendra Kumari ${ }^{1 *}$, K.K. Bhardwaj ${ }^{1}$, \\ Ashwani Kumar ${ }^{2}$ and Tarun Kumar ${ }^{1}$ \\ ${ }^{1}$ Department of Forestry, CCS Haryana Agricultural University, Hisar-125004, Haryana, India \\ ${ }^{2}$ Department of Plant Pathology, CCS Haryana Agricultural University, \\ Hisar-125004, Haryana, India \\ *Corresponding author
}

\section{A B S T R A C T}

The study was conducted to explore the growth performance of the 19 promising clones of Populus deltoides at nursery stage. Data were statistically analyzed for different growth parameters viz., sprouting percentage, colour and shape of buds (after one month), total height, collar diameter, inter-nodal length, number of roots, number of branches, root

\section{Keywords}

Populus deltoides clones, Growth parameters, Nursery.

\section{Article Info}

Accepted: 15 October 2017 Available Online: 10 December 2017 length, volume index, total biomass (fresh shoot + fresh root weight), bark colour and texture, disease resistance (after six months and one year), number of leaves, leaf fall duration, leaf length, leaf width and leaf area index at nursery stage. Taxonomic evaluation (colour and shape of buds) revealed that the clonal characters further represent the source of germplasm from Populus deltoides of section Aigeirous. Results revealed that clone W39 showed significantly better performance for total height, collar diameter, number of branches, number of roots, volume index and biomass. Clone W32 had more inter-nodal length and root length in comparison to other clones. Clones Udai, G48, W110, $\mathrm{S}_{7} \mathrm{C}_{8}$, Bahar, $\mathrm{S}_{7} \mathrm{C}_{1}$ were also found to be the promising clones for growth parameters. Clones from Wimco seedlings ltd. showed better performance for all above mentioned growth characters in comparison to FRIAM clones. Leaf parameters were found to vary in all 19 clones. In this study it was also observed that clones varied for disease resistance, bark colour and texture (mainly dark greenish bark colour showed disease resistance at one year stage). The correlation results indicated the feasibility of selection of clones for higher values of total height, collar diameter, root length and volume index.

\section{Introduction}

Poplar (Populus deltoides) a short-rotation commercial agroforestry tree, has received wide acceptance during the last three decades in India due to its fast growth habit, its compatibility with agriculture crops, and high industrial requirements. The species is widely grown in Indo-Gangetic region of the country comprising Uttarakhand, Uttar Pradesh, Punjab, Haryana, Delhi, Jammu and Kashmir also in some pockets of Bihar and Madhya Pradesh distributed on 312,000 ha out of which $60 \%$ is block plantation and $40 \%$ is bund plantation (ICFRE, 2012). The tree is harvested at a short rotation of 6-8 years, which provides a yield of $150-200 \mathrm{~m}^{3}$ per ha (mean annual increment of $20-25 \mathrm{~m}^{3}$ per ha per year) in block plantation and $12-20 \mathrm{~m}^{3}$ per ha (mean annual increment of $2-3 \mathrm{~m}^{3}$ per ha 
per year) in boundary plantations (Kishwan and Kumar, 2013). The wood of the tree is mainly used for plywood manufacturing in India. The branches, tops and roots of the trees are also used by plywood industries as a fuel, which helps reduce fossil fuel use. Due to its fast growth and wider adoptability, the tree has huge potential to sequester carbon and mitigate $\mathrm{CO}_{2}$ from the atmosphere (Dhiman, 2009; Singh and Lodhiyal, 2009; Chauhan et al., 2010 and Gera, 2012).

Poplar was introduced in India in 1950. $P$. deltoides grows better on well-drained fertile soils with a neutral soil pH. Farmers have started raising poplar on a large scale as block/boundary plantations in the states of Punjab, Haryana, Uttar Pradesh, Uttrakhand and Himachal Pradesh, but poplar plantations in these states have a narrow genetic base consisting of only a few superior clones.

Keeping in view the ever increasing demand of poplar wood and the interest of farmers in cultivation of poplar, different promising clones have been developed by various research organisations in the country and are being tested in a number of locations under "All India coordinated research project on poplar improvement" (Kumar et al., 1999; Singh et al., 1999).

Poplar research is facing problems regarding mixing of clones. Forestry unlike agriculture is a long term proposition and mistakes committed once are reflected after several years or even recognized due to nonidentification of the different clones. Identification of different clones of poplar on the basis of morphological characters is nearly impossible due to lack of visible and contrasting traits among the different clones. But identification of different clones is very necessary for the maintenance of purity at nursery and plantation stages. Poplar is a deciduous species, therefore leaf related characters are available in the winter months while vegetative buds remain in stable state during that whole period. Therefore present study was carried out to understand morphological growth parameter and biomass production of different clones in Harayana for future tree improvement program,

\section{Materials and Methods}

The present investigation was carried out in experimental field of Department of Forestry, CCS HAU, Hisar $\left(29^{\circ} 10^{\prime} \mathrm{N}\right.$ latitude and $75^{\circ}$ $46^{\prime} \mathrm{E}$ longitude at an elevation of $215.2 \mathrm{~m}$ above mean sea level, Mean annual minimum and maximum temperature is $16.2^{\circ} \mathrm{C}$ and $31.5^{\circ} \mathrm{C}$, respectively). Hisar has a typical semi-arid climate with hot and dry summer and extremely cold winter. The soil at the planting site is sandy loam with an average $\mathrm{pH}$ of 8.5. Healthy vigorous and disease free cuttings $(20-22 \mathrm{~cm}$ in length and $1-1.5 \mathrm{~cm}$ in thickness) of nineteen clones (Table 1) obtained from forest nurseries of four districts Yamunanagar, Karnal, Kurukshetra and Bithmada (Hisar) of Haryana state were planted at $60 \times 80 \mathrm{~cm}$ in second week of February following randomized block design with three replications with standard package of practice. Six plants of each clone from each of the three replications were selected randomly for recording data on total height, collar diameter, inter-nodal length, number of roots, number of branches, root length, volume index, total biomass (fresh shoot + fresh root weight), bark colour and texture, disease resistance (after six months and one year), number of leaves, leaf fall duration, leaf length, leaf width and leaf area index at nursery stage whereas the sprouting percentage, colour and shape of buds were observed after one month plantation of cuttings in the nursery. Leaf area index and volume index were estimated as per Chauhan (2008). The replicated data recorded for all characters was analyzed statistically (Panse and Sukhatme, 1978). Significant differences were based on $\mathrm{P} \leq 0.05$. 


\section{Results and Discussion}

Studies indicated that taxonomical buds of different clones varied with regard to position shape, colour and orientation. The data on initiation of sprouting of cuttings was recorded. It was observed that sprouting percentage was maximum $(93 \%)$ in W39, W108, $\mathrm{S}_{7} \mathrm{C}_{8}$, Bahar and FRIAM 70 followed by W22, $\mathrm{S}_{7} \mathrm{C}_{1}$, FRIAM 100, FRIAM 118 , FRIAM 81, G48, Udai.

The clone FRIAM 107 showed the lowest sprouting percentage (75\%). Poplar clones viz., G48, Bahar, FRIAM 100, FRIAM 118, FRIAM 37, FRIAM 107, FRIAM 81, FRIAM $72, \mathrm{~S}_{7} \mathrm{C}_{1}$ had similar shape (short terminal and narrow conical in shape) of buds but arrangement on stems is different. WIMCO seedlings ltd. clones, FRIAM $70, \mathrm{~S}_{7} \mathrm{C}_{8}$ had similar shape (long terminal and narrow conical) but out of these clones FRIAM 100, FRIAM 118, Bahar, FRIAM 107, FRIAM 72 had broad ovoid in shape. Clones also varied for bud colour. Greenish brown colour of buds was observed in clones W39, Udai, W109, FRIAM 107, FRIAM 81, FRIAM 118, Bahar, FRIAM 70, $\mathrm{S}_{7} \mathrm{C}_{8}$ and light greenish brown colour was observed in clones W110, FRIAM 100, FRIAM 37, FRIAM 72.

Clones G48, W108, W22, W32, WSL 22 had red brown colour of buds and light red brown colour was observed in W110, $\mathrm{S}_{7} \mathrm{C}_{1}$. Taxonomic studies made on various feature of buds revealed that the various selected clones with ambigious identity fell under the section aigerious and the taxa belong to Populus deltoides (Table 2). Also the studies on winter buds of different clones of Populus deltoides varied with regard to various characters and it can be used as a morphological marker to distinguish clones (Alpana and Biswas, 1999). Chaudhary and Tewari (2006) also reported same results in twenty different clones through morphological marker.
In this study, a lot of variation was observed in growth parameters of different Populus deltoides clones, viz., total height (2.88-5.17 $\mathrm{m})$, collar diameter $(2.05-2.71 \mathrm{~cm})$, internodal length $(4.40-5.80 \mathrm{~cm})$, number of branches (8.03-13.60), number of roots (10.50-20.00), root length $(49.30-80.00 \mathrm{~cm})$, volume index (950.09-2959.56 $\left.\mathrm{cm}^{3}\right)$, total biomass (960-3250 g) after one year, total height (1.99-3.82 m), collar diameter (1.59$2.20 \mathrm{~cm})$, inter-nodal length $(2.86-4.01 \mathrm{~cm})$, number of branches (6.36-10.60), number of roots (7.15-15.30), root length (28.70-50.40 $\mathrm{cm})$, volume index $\left(401.87-1390.95 \mathrm{~cm}^{3}\right)$ and total biomass (680-2170 g) after six months. A lot of variation was also found in leaf parameters (Table 3) viz., number of leaves (110-290), leaf fall duration (80-110 in days), leaf width $(13-18 \mathrm{~cm})$, leaf length (14.5-21 $\mathrm{cm})$ and leaf area index (188.5-378). In this study, it was observed that clones W39, Udai, G48 overtook all other clones and showed maximum height growth, collar diameter, number of branches, number of roots, volume index and total biomass. Clones W32 and Udai had maximum inter-nodal length, whereas clones W32, G48, $\mathrm{S}_{7} \mathrm{C}_{8}, \mathrm{~W} 39$ had maximum root length than other clones at data recorded after six months and after one year.

Other clones viz., W110, W22, W109, $\mathrm{S}_{7} \mathrm{C}_{1}$, Bahar, $\mathrm{S}_{7} \mathrm{C}_{8}$ were considered significantly promising clones under. Clones FRIAM 81, FRIAM 37 had poor performance as compared to other clones. The poor performance in all the clones has been due to non-application of any fertilizer to the soil or due to their genetic make-up. It was observed from the Table 3 and 4 that all Wimco seedlings ltd. clones showed significantly better growth performance in the nursery over other clones. Various workers had also studied the performance of Populus deltoides clones under nursery conditions and reported similar results. 
Table.1 Different clones of poplar (Populus deltoides) used in this study

\begin{tabular}{|l|l|l|l|l|l|}
\hline Sr.no. & Poplar clones & $\begin{array}{l}\text { Place of } \\
\text { origin }\end{array}$ & Sr.no. & Poplar clones & Place of origin \\
\hline $\mathbf{1}$ & WSL 22 & $\begin{array}{l}\text { WIMCO \& UP } \\
\text { Forest } \\
\text { Department }\end{array}$ & $\mathbf{1 1}$ & FRIAM 100 & USA \\
\hline $\mathbf{2}$ & FRIAM 72 & USA & $\mathbf{1 2}$ & W 109 & \\
\hline $\mathbf{3}$ & $\mathrm{S}_{7} \mathrm{C}_{8}$ & Texas, USA & $\mathbf{1 3}$ & W32 & \\
\hline $\mathbf{4}$ & FRIAM 70 & FRI, Dehradun & $\mathbf{1 4}$ & Udai & WIMCO (G48×G3) \\
\hline $\mathbf{5}$ & FRIAM 81 & USA & $\mathbf{1 5}$ & W 22 & WIMCO \\
\hline $\mathbf{6}$ & FRIAM 107 & USA & $\mathbf{1 6}$ & W 108 & WIMCO \\
\hline $\mathbf{7}$ & Bahar & WIMCO & $\mathbf{1 7}$ & W39 & WIMCO \\
\hline $\mathbf{8}$ & FRIAM 37 & USA & $\mathbf{1 8}$ & W110 & WIMCO \\
\hline $\mathbf{9}$ & S $_{7} \mathrm{C}_{1}$ & Texas, USA & $\mathbf{1 9}$ & G48 & Texas, USA \\
\hline $\mathbf{1 0}$ & FRIAM 118 & USA & & & \\
\hline
\end{tabular}

Table.2 Sprouting percentage and morphological characters (colour and shape) as exhibited by buds in different clones of Populus deltoides after one month of planting of cuttings

\begin{tabular}{|l|l|l|l|}
\hline $\begin{array}{l}\text { Poplar } \\
\text { clones }\end{array}$ & $\begin{array}{l}\text { Sprouting } \\
\text { \% }\end{array}$ & Colour of buds & Shape of buds \\
\hline G48 & 87 & Red Brown & Short terminal, narrow conical in shape \\
\hline W110 & 80 & Lightly red brown & Long terminal, narrow conical in shape \\
\hline W39 & 93 & Greenish Brown & Long terminal, narrow conical in shape \\
\hline W108 & 93 & Red brown & Long terminal and narrow conical in shape \\
\hline W22 & 92 & Red Brown & Long terminal and conical in shape \\
\hline Udai & 87 & Greenish Brown & $\begin{array}{l}\text { Long terminal, narrow conical in shape on } \\
\text { upper portion bud's tip is curved out }\end{array}$ \\
\hline W32 & 81 & Red brown & Long terminal and narrow conical in shape \\
\hline W109 & 87 & Greenish brown & Bud is Long terminal. \\
\hline FRIAM 100 & 91 & Light greenish brown & Bud is short and ovoid in shape \\
\hline FRIAM 118 & 90 & Greenish brown & Bud is short and broad ovoid shape \\
\hline S C $_{1}$ & 90 & Lightly red brown & $\begin{array}{l}\text { Short and narrow conical in shape, on } \\
\text { upper portion bud's tip is curved out }\end{array}$ \\
\hline FRIAM 37 & 81 & Light greenish brown & $\begin{array}{l}\text { Short terminal and Narrow conical in } \\
\text { shape }\end{array}$ \\
\hline Bahar & 93 & Greenish brown & Bud is small and ovoid in shape. \\
\hline FRIAM 107 & 75 & Greenish brown & Bud is short terminal and ovoid in shape \\
\hline FRIAM 81 & 92 & Greenish brown & Bud is short terminal. \\
\hline FRIAM 70 & 93 & Greenish brown & long terminal and narrow conical in shape \\
\hline S7 C $_{8}$ & 93 & Greenish brown & Long terminal and narrow conical in shape \\
\hline FRIAM 72 & 81 & Light greenish brown & Short terminal and broad ovoid in shape \\
\hline WSL 22 & 87 & Red brown & Long terminal and conical in shape \\
\hline
\end{tabular}


Table.3 Growth performance of six months old Populus deltoides clones in nursery

\begin{tabular}{|c|c|c|c|c|c|c|c|c|}
\hline $\begin{array}{l}\text { Poplar } \\
\text { Clones }\end{array}$ & $\begin{array}{l}\text { Height } \\
\text { (m) }\end{array}$ & $\begin{array}{c}\text { Collar } \\
\text { diameter } \\
(\mathrm{cm})\end{array}$ & $\begin{array}{c}\text { Internodal } \\
\text { length } \\
(\mathrm{cm})\end{array}$ & $\begin{array}{c}\text { No. of } \\
\text { branches }\end{array}$ & $\begin{array}{l}\text { No. of } \\
\text { roots }\end{array}$ & $\begin{array}{c}\text { Root } \\
\text { length } \\
(\mathrm{cm})\end{array}$ & $\begin{array}{c}\text { Volume } \\
\text { index } \\
\left(\mathrm{cm}^{3}\right)\end{array}$ & $\begin{array}{c}\text { Total } \\
\text { biomass }(\text { g) } \\
(\text { FSW+FRW) }\end{array}$ \\
\hline G48 & $3.57(2)$ & $2.02(4)$ & $3.37(8)$ & $8.60(6)$ & $9.63(9)$ & $47.40(2)$ & $1153.46(3)$ & $1860(2)$ \\
\hline W110 & $3.43(5)$ & $1.99(7)$ & $2.94(18)$ & $7.30(15)$ & $9.40(10)$ & $40.30(8)$ & $1078.04(8)$ & $1520(4)$ \\
\hline W39 & $3.82(1)$ & $2.20(1)$ & $3.84(4)$ & $10.60(1)$ & $15.30(1)$ & $44.50(3)$ & $1390.95(1)$ & $2170(1)$ \\
\hline W108 & $3.06(9)$ & $1.90(12)$ & $3.11(14)$ & $7.95(9)$ & $12.62(3)$ & $41.10(7)$ & $886.50(10)$ & $990(14)$ \\
\hline W22 & $3.16(8)$ & $2.07(3)$ & $2.86(19)$ & $7.14(16)$ & $8.80(13)$ & $34.30(13)$ & $1081.85(6)$ & $1170(9)$ \\
\hline Udai & $3.57(3)$ & $2.11(2)$ & $3.96(2)$ & $7.81(12)$ & $11.20(5)$ & $39.70(10)$ & $1258.97(2)$ & $1380(5)$ \\
\hline W32 & $2.81(13)$ & $1.80(16)$ & $4.01(1)$ & $9.80(3)$ & $9.90(7)$ & $50.40(1)$ & $743.75(13)$ & $1130(10)$ \\
\hline W109 & $2.95(11)$ & $1.93(10)$ & $3.13(12)$ & $7.08(17)$ & $13.20(2)$ & $38.80(11)$ & $871.26(11)$ & $1700(3)$ \\
\hline FRIAM100 & $2.24(15)$ & $1.74(17)$ & $2.96(17)$ & $8.30(8)$ & $8.27(16)$ & $30.10(17)$ & 538.96(17) & $1010(13)$ \\
\hline FRIAM118 & $2.13(17)$ & $1.86(14)$ & $3.31(10)$ & $7.90(10)$ & $8.40(15)$ & $33.60(15)$ & $591.52(16)$ & $970(16)$ \\
\hline $\mathrm{S}_{7} \mathrm{C}_{1}$ & $3.33(7)$ & $1.99(6)$ & $3.28(11)$ & $8.50(7)$ & $8.90(12)$ & $43.40(4)$ & $1150.03(4)$ & $1250(8)$ \\
\hline FRIAM 37 & $1.99(19)$ & $1.65(18)$ & $3.12(13)$ & $7.70(13)$ & $10.88(6)$ & $29.90(18)$ & $475.58(18)$ & $830(18)$ \\
\hline Bahar & $3.46(4)$ & $1.98(8)$ & $3.90(3)$ & $9.56(4)$ & $8.96(11)$ & $41.10(6)$ & $1078.76(7)$ & $1070(12)$ \\
\hline FRIAM107 & $2.22(16)$ & $1.92(11)$ & $3.01(16)$ & $6.45(18)$ & $7.81(18)$ & $33.10(16)$ & $640.21(15)$ & $840(17)$ \\
\hline FRIAM 81 & $2.00(18)$ & $1.59(19)$ & $3.35(9)$ & $6.36(19)$ & $8.74(14)$ & $28.70(19)$ & $401.87(19)$ & $680(19)$ \\
\hline FRIAM 70 & $2.90(12)$ & $1.88(13)$ & $3.65(7)$ & $7.35(14)$ & $8.03(17)$ & $39.80(9)$ & $814.21(12)$ & $1310(7)$ \\
\hline $\mathrm{S}_{7} \mathrm{C}_{8}$ & $3.33(6)$ & $2.00(5)$ & $3.80(5)$ & $7.85(11)$ & $11.27(4)$ & $43.00(5)$ & $1128.12(5)$ & $1340(6)$ \\
\hline FRIAM 72 & $2.49(14)$ & $1.84(15)$ & $3.78(6)$ & $9.90(2)$ & $7.15(19)$ & $34.00(14)$ & $667.71(14)$ & $980(15)$ \\
\hline WSL 22 & $3.00(10)$ & $1.95(9)$ & $3.03(15)$ & $9.03(5)$ & $9.80(8)$ & $38.60(12)$ & 904.04(9) & $1090(11)$ \\
\hline Range & $\begin{array}{l}1.99- \\
3.82\end{array}$ & $1.59-2.20$ & $2.86-4.01$ & $\begin{array}{l}6.36- \\
10.60 \\
\end{array}$ & $\begin{array}{l}7.15- \\
15.30 \\
\end{array}$ & $\begin{array}{l}28.70- \\
50.40\end{array}$ & $\begin{array}{l}401.87- \\
1390.95 \\
\end{array}$ & $680-2170$ \\
\hline $\mathrm{CD}(\mathrm{p}=0.05)$ & 0.21 & 0.07 & 0.14 & 0.85 & 0.58 & 4.25 & 110.71 & 135.44 \\
\hline
\end{tabular}

(Value in parentheses indicate ranking), FSW- Fresh Shoot Weight, FRW- Fresh Root Weight

Table.4 Growth performance of one-year old Populus deltoides clones in nursery

\begin{tabular}{|c|c|c|c|c|c|c|c|c|}
\hline $\begin{array}{l}\text { Poplar } \\
\text { Clones }\end{array}$ & $\begin{array}{l}\text { Height } \\
\text { (m) }\end{array}$ & $\begin{array}{c}\text { Collar } \\
\text { diameter } \\
(\mathrm{cm})\end{array}$ & $\begin{array}{l}\text { Internodal } \\
\text { length } \\
(\mathrm{cm})\end{array}$ & $\begin{array}{c}\text { No. of } \\
\text { branches }\end{array}$ & $\begin{array}{l}\text { No. of } \\
\text { roots }\end{array}$ & $\begin{array}{l}\text { Root } \\
\text { length } \\
(\mathrm{cm})\end{array}$ & $\begin{array}{c}\text { Volume } \\
\text { index } \\
\left(\mathrm{cm}^{3}\right)\end{array}$ & $\begin{array}{c}\text { Total } \\
\text { biomass (g) } \\
(\text { FSW+FRW) }\end{array}$ \\
\hline G48 & $4.76(3)$ & $2.55(3)$ & $4.95(9)$ & $10.43(10)$ & $14.57(8)$ & $74.20(2)$ & $2482.35(3)$ & $2850(2)$ \\
\hline W110 & $4.43(5)$ & $2.40(9)$ & $4.53(18)$ & $9.83(12)$ & $14.18(9)$ & $69.10(6)$ & $2225.63(4)$ & $2270(4)$ \\
\hline W39 & $5.17(1)$ & $2.71(1)$ & $5.26(5)$ & $13.60(1)$ & $20.00(1)$ & $71.20(4)$ & $2959.56(1)$ & $3250(1)$ \\
\hline W108 & $4.13(9)$ & $2.34(13)$ & $4.54(17)$ & $10.10(11)$ & $18.66(3)$ & $66.50(9)$ & $1763.17(10)$ & $1430(14)$ \\
\hline W22 & $4.30(8)$ & $2.46(6)$ & $4.64(15)$ & $9.59(14)$ & $12.61(12)$ & $65.80(10)$ & $2042.71(8)$ & $1670(9)$ \\
\hline Udai & $4.88(2)$ & $2.62(2)$ & $5.62(2)$ & $10.54(8)$ & $16.33(5)$ & $68.30(8)$ & $2525.83(2)$ & $2100(6)$ \\
\hline W32 & $3.93(12)$ & $2.38(11)$ & $5.80(1)$ & $12.60(2)$ & $14.70(7)$ & $80.00(1)$ & $1747.49(11)$ & $1470(11)$ \\
\hline W109 & $4.12(10)$ & $2.40(10)$ & $4.70(14)$ & $9.30(16)$ & $19.20(2)$ & $69.10(7)$ & $1862.89(9)$ & $2640(3)$ \\
\hline FRIAM100 & $3.36(15)$ & $2.19(17)$ & $4.40(19)$ & $11.08(5)$ & $12.43(15)$ & $52.00(17)$ & $1265.03(16)$ & $1450(13)$ \\
\hline FRIAM118 & $3.28(1)$ & $2.32(14)$ & $4.90(10)$ & $10.46(9)$ & $12.36(1)$ & $58.30(15)$ & $1385.86(14)$ & $1360(16)$ \\
\hline $\mathrm{S}_{7} \mathrm{C}_{1}$ & $4.31(7)$ & $2.51(4)$ & $4.88(11)$ & $10.96(6)$ & $13.26(11)$ & $70.60(5)$ & $2131.53(6)$ & $1900(8)$ \\
\hline FRIAM 37 & $3.01(18)$ & $2.08(18)$ & $4.75(13)$ & $9.20(17)$ & $16.63(4)$ & $49.80(18)$ & $1022.26(18)$ & $1110(18)$ \\
\hline Bahar & $4.41(6)$ & $2.45(7)$ & $5.20(6)$ & $12.01(3)$ & $12.46(14)$ & $62.60(12)$ & $2077.98(7)$ & $1370(15)$ \\
\hline FRIAM107 & $3.22(17)$ & $2.32(15)$ & $4.60(16)$ & $8.49(18)$ & $10.50(19)$ & $53.00(16)$ & $1360.50(15)$ & $1120(17)$ \\
\hline FRIAM 81 & $2.88(19)$ & $2.05(19)$ & $5.00(8)$ & $8.03(19)$ & $11.69(17)$ & 49.30(19) & $950.09(19)$ & $960(19)$ \\
\hline FRIAM 70 & $3.86(13)$ & $2.38(12)$ & $5.10(7)$ & $9.45(15)$ & $12.55(13)$ & $63.10(11)$ & $1716.36(12)$ & $1924(7)$ \\
\hline $\mathrm{S}_{7} \mathrm{C}_{8}$ & $4.44(4)$ & $2.50(5)$ & $5.33(3)$ & $9.78(13)$ & $15.60(6)$ & $72.40(3)$ & $2178.37(5)$ & $2250(5)$ \\
\hline FRIAM 72 & $3.40(14)$ & $2.20(16)$ & $5.30(4)$ & $11.98(4)$ & $10.65(18)$ & $61.10(13)$ & $1257.60(17)$ & $1470(12)$ \\
\hline WSL 22 & $3.97(11)$ & $2.40(8)$ & $4.80(12)$ & $10.96(7)$ & $14.13(10)$ & $58.40(14)$ & $1659.42(13)$ & $1650(10)$ \\
\hline Range & $\begin{array}{l}2.88- \\
5.17\end{array}$ & $2.05-2.71$ & $4.40-5.80$ & $\begin{array}{l}8.03- \\
13.60\end{array}$ & $\begin{array}{l}10.50- \\
20.0\end{array}$ & $\begin{array}{l}49.30- \\
80.00\end{array}$ & $\begin{array}{l}950.09- \\
2959.56\end{array}$ & $960-3250$ \\
\hline $\mathrm{CD}(\mathrm{p}=0.05)$ & 0.39 & 0.06 & 0.35 & 1.10 & 1.18 & 4.48 & 137.76 & 171.16 \\
\hline
\end{tabular}

(Value in parentheses indicate ranking), FSW- Fresh Shoot Weight, FRW- Fresh Root Weight 
Table.5 Morphological characters of different clones of poplar at six month, one year nursery stage

\begin{tabular}{|l|l|l|c|l|}
\hline \multirow{2}{*}{ Clones } & \multicolumn{2}{|c|}{ Six month stage } & \multicolumn{1}{c|}{ One year stage } \\
\cline { 2 - 5 } & $\begin{array}{c}\text { Disease } \\
\text { resistance }\end{array}$ & \multicolumn{1}{|c|}{ Bark colour and texture } & $\begin{array}{c}\text { Disease } \\
\text { resistance }\end{array}$ & Bark colour and texture \\
\hline G48 & Yes & light greenish, smooth & No & Light White spots to green, less smooth \\
\hline W110 & No & Dark Greenish, smooth & No & White spots to Dark green, smooth \\
\hline W39 & Yes & Dark Greenish, smooth & Yes & White spots to Dark green, less smooth \\
\hline W108 & Yes & Dark Greenish, smooth & Yes & White spots to dark green, smooth \\
\hline W22 & Yes & Dark Greenish, smooth & Yes & White spots to dark greenish, smooth \\
\hline Udai & Yes & Light Greenish, smooth & Yes & White spots to dark greenish, smooth \\
\hline W32 & No & Light Greenish, smooth & No & Light White spots to green, less smooth \\
\hline W109 & Yes & Light Greenish, less smooth & Yes & Light white spots to green, less smooth \\
\hline FRIAM 100 & No & Light Greenish, less smooth & No & Light white spots to green. less smooth \\
\hline FRIAM 118 & No & Light Greenish, less smooth & No & White spots to dark green, less smooth \\
\hline S C $_{1}$ & Yes & Dark Greenish, smooth & No & Light White spots to greenish, smooth \\
\hline FRIAM 37 & No & Light Greenish, smooth & No & Light white spots to green, less smooth \\
\hline Bahar & No & Light Greenish, less smooth & No & Light white spots to green, less smooth \\
\hline FRIAM 107 & No & Light Greenish, smooth & No & Light white spots to green, smooth \\
\hline FRIAM 81 & No & light greenish, less smooth & No & Light white spots to green, less smooth \\
\hline & Yes & Dark Greenish, smooth & Yes & $\begin{array}{l}\text { White spots to dark greenish, less } \\
\text { smooth }\end{array}$ \\
\hline FRIAM 70 & Yes & light Greenish, smooth & No & Light white spots to green, smooth \\
\hline S C ${ }_{8}$ & Yes & Dark Greenish, less smooth & No & $\begin{array}{l}\text { White spots to dark greenish, less } \\
\text { smooth }\end{array}$ \\
\hline FRIAM 72 & Yes & Light Greenish, less smooth & Yes & White spots to green, less smooth \\
\hline WSL 22 & & & \\
\hline
\end{tabular}

Table.6 Leaf parameters of different clones of Populus deltoides in the nursery

\begin{tabular}{|l|l|l|l|l|l|}
\hline \multicolumn{1}{|c|}{ Clones } & $\begin{array}{c}\text { No. of leaves } \\
\text { per plant }\end{array}$ & $\begin{array}{c}\text { Leaf fall duration } \\
\text { (days) }\end{array}$ & $\begin{array}{c}\text { Leaf width } \\
(\mathbf{c m})\end{array}$ & $\begin{array}{c}\text { Leaf length } \\
(\mathbf{c m})\end{array}$ & Leaf area index \\
\hline G48 & $238(4)$ & $90-100$ & 16.0 & 16.5 & $264.00(7)$ \\
\hline W110 & $180(13)$ & $80-90$ & 17.0 & 17.5 & $297.50(4)$ \\
\hline W39 & $290(1)$ & $80-90$ & 15.0 & 17.0 & $255.00(9)$ \\
\hline W108 & $170(15)$ & $90-110$ & 15.0 & 16.5 & $247.50(10)$ \\
\hline W22 & $204(8)$ & $90-95$ & 14.5 & 16.0 & $232.00(14)$ \\
\hline Udai & $230(5)$ & $90-110$ & 14.0 & 15.5 & $217.00(17)$ \\
\hline W32 & $245(2)$ & $90-90$ & 15.0 & 17.5 & $262.50(8)$ \\
\hline W 109 & $190(10)$ & $90-100$ & 15.5 & 18.0 & $279.00(6)$ \\
\hline FRIAM 100 & $210(7)$ & $90-100$ & 14.5 & 16.5 & $239.25(13)$ \\
\hline FRIAM 118 & $187(11)$ & $100-110$ & 15.0 & 16.0 & $240.00(12)$ \\
\hline S $_{7} C_{1}$ & $110(19)$ & $90-95$ & 14.0 & 16.0 & $224.00(15)$ \\
\hline FRIAM 37 & $160(16)$ & $100-110$ & 14.5 & 15.0 & $217.50(16)$ \\
\hline Bahar & $220(6)$ & $90-95$ & 18.0 & 21.0 & $378.00(1)$ \\
\hline FRIAM 107 & $145(17)$ & $90-100$ & 13.0 & 14.5 & $188.50(19)$ \\
\hline FRIAM 81 & $185(12)$ & $85-95$ & 13.0 & 15.5 & $201.50(18)$ \\
\hline FRIAM 70 & $240(3)$ & $90-100$ & 14.5 & 17.0 & $246.50(11)$ \\
\hline$S_{7} C_{8}$ & $145(18)$ & $90-100$ & 15.5 & 19.0 & $294.50(5)$ \\
\hline FRIAM 72 & $173(14)$ & $90-110$ & 17.0 & 20.5 & $348.50(3)$ \\
\hline WSL 22 & $195(9)$ & $90-95$ & 17.0 & 21 & $357.00(2)$ \\
\hline Range & $110-290$ & $80-110$ & $13-18$ & $14.5-21$ & $188.50-378.00$ \\
\hline CD (p=0.05) & 30.94 & ------- & 0.95 & 0.98 & 8.76 \\
\hline
\end{tabular}

(Value in parentheses indicate ranking). 
Table.7 Correlation among phenotypic characters of different clones of Populus deltoids

\begin{tabular}{|c|c|c|c|c|c|c|c|c|}
\hline Characters & $\begin{array}{c}\text { Data } \\
\text { recorded }\end{array}$ & $\begin{array}{c}\text { Total } \\
\text { height }(m)\end{array}$ & $\begin{array}{c}\text { Collar } \\
\text { diameter }(\mathbf{c m})\end{array}$ & $\begin{array}{r}\text { Internodal } \\
\text { length }(\mathrm{cm})\end{array}$ & $\begin{array}{c}\text { No. of } \\
\text { branches }\end{array}$ & $\begin{array}{l}\text { No. of } \\
\text { roots }\end{array}$ & $\begin{array}{c}\text { Root } \\
\text { length }(\mathrm{cm})\end{array}$ & $\begin{array}{c}\text { Volume } \\
\text { index }\left(\mathrm{cm}^{3}\right)\end{array}$ \\
\hline $\begin{array}{c}\text { Total height } \\
(\mathrm{m})\end{array}$ & $\begin{array}{l}\mathrm{T} 1 \\
\mathrm{~T} 2\end{array}$ & $\begin{array}{l}1 \\
1\end{array}$ & & & & & & \\
\hline $\begin{array}{l}\text { Collar } \\
\text { diameter }(\mathrm{cm})\end{array}$ & $\begin{array}{l}\mathrm{T} 1 \\
\mathrm{~T} 2\end{array}$ & $\begin{array}{l}0.873 * * \\
0.939 * *\end{array}$ & $\begin{array}{l}1 \\
1\end{array}$ & & & & & \\
\hline $\begin{array}{l}\text { Internodal } \\
\text { length }(\mathrm{cm})\end{array}$ & $\begin{array}{l}\text { T1 } \\
\text { T2 }\end{array}$ & $\begin{array}{l}0.342 \\
0.305 \\
\end{array}$ & $\begin{array}{l}0.209 \\
0.345 \\
\end{array}$ & $\begin{array}{l}1 \\
1 \\
\end{array}$ & & & & \\
\hline $\begin{array}{c}\text { No. of } \\
\text { branches }\end{array}$ & $\begin{array}{l}\mathrm{T} 1 \\
\mathrm{~T} 2\end{array}$ & $\begin{array}{l}0.390 \\
0.465 *\end{array}$ & $\begin{array}{l}0.299 \\
0.460 *\end{array}$ & $\begin{array}{l}0.566 * * \\
0.488^{*}\end{array}$ & $\begin{array}{l}1 \\
1\end{array}$ & & & \\
\hline No. of roots & $\begin{array}{l}\text { T1 } \\
\text { T2 }\end{array}$ & $\begin{array}{l}0.473^{*} \\
0.532^{*}\end{array}$ & $\begin{array}{l}0.408 \\
0.426 \\
\end{array}$ & $\begin{array}{l}0.167 \\
0.077 \\
\end{array}$ & $\begin{array}{l}0.227 \\
0.211 \\
\end{array}$ & $\begin{array}{l}1 \\
1 \\
\end{array}$ & & \\
\hline $\begin{array}{c}\text { Root } \\
\text { length }(\mathrm{cm})\end{array}$ & $\begin{array}{l}\mathrm{T} 1 \\
\mathrm{~T} 2 \\
\end{array}$ & $\begin{array}{l}0.896 * * \\
0.790 * * \\
\end{array}$ & $\begin{array}{l}0.776 * * \\
0.753 * * \\
\end{array}$ & $\begin{array}{l}0.487 * \\
0.480 *\end{array}$ & $\begin{array}{l}0.346 \\
0.465 *\end{array}$ & $\begin{array}{l}0.451 * \\
0.440 *\end{array}$ & $\begin{array}{l}1 \\
1 \\
\end{array}$ & \\
\hline $\begin{array}{c}\text { Volume } \\
\text { index }\left(\mathrm{cm}^{3}\right)\end{array}$ & $\begin{array}{l}\mathrm{T} 1 \\
\mathrm{~T} 2\end{array}$ & $\begin{array}{l}0.977 * * \\
0.985 * *\end{array}$ & $\begin{array}{l}0.936 * * \\
0.960 * *\end{array}$ & $\begin{array}{l}0.308 \\
0.307\end{array}$ & $\begin{array}{l}0.353 \\
0.452 *\end{array}$ & $\begin{array}{l}0.480 * \\
0.504 *\end{array}$ & $\begin{array}{l}0.875 * * \\
0.772 * *\end{array}$ & $\begin{array}{l}1 \\
1\end{array}$ \\
\hline
\end{tabular}

*Significant at 5 per cent level of significance, ** Significant at 1 per cent level of significance, T1 =after 6 month, T2 = after 1 year.

Verma and Bangarwa (2003) suggested that WIMCO seedlings ltd. clones had better growth characters than LalKuan clones. Verma (2001) studied the performance of poplar clones under nursery conditions and reported similar results. Toky et al., (1996) also studied the performance of various clones and observed a lot of variation in the growth parameters of different Populus deltoides clones. Rawat et al., (2001) earmarked eleven clones as best and twelve as potential clones on the basis of nursery screening. Similar results was also reported by Karnatka and Chandra (1995) that growth performance of five clones, viz., Udai, Kranti, Bahar, 72/58 and St-74 had come close to clone G48 and clone Udai and Bahar had shown more promise than Kranti.

\section{Disease resistance}

Disease resistance (from yellowing, black leaf spots and crumpled of leaves) and bark colour and texture was observed visually abd presented in Table 5 and 6. Poplar clones of G48, W39, W108, W22, Udai, W109, $\mathrm{S}_{7} \mathrm{C}_{1}$, $\mathrm{S}_{7} \mathrm{C}_{8}$, FRIAM 70, FRIAM 72 and WSL 22 showed disease resistance after six months while others are found to be susceptible. But after one year only clone W39, W108, W22, Udai, W109, FRIAM 70 and WSL 22 showed disease resistance. The susceptibility of clones to different disease may be due to weather. Clones varied in bark colour and texture.

Clones G48, W32, W109, FRIAM 100, Bahar, $\mathrm{S}_{7} \mathrm{C}_{1}$, FRIAM 37, FRIAM 107, $\mathrm{S}_{7} \mathrm{C}_{8}$ and FRIAM 81 had similar bark colour and texture (light white spots to greenish, less smooth) but clones S7C1, FRIAM 107, $\mathrm{S}_{7} \mathrm{C}_{8}$ had smooth bark and clones W110, W39, 108, Udai, WSL 22, FRIAM 72, FRIAM 70 and FRIAM 118 had similar shape (white spots to dark greenish, less smooth) but clones W110, W108, Udai had smooth bark after one year.

Clones G48, Udai, W32, W109, FRIAM 100. FRIAM 118, FRIAM 37, FRIAM 107, FRIAM 81, $\mathrm{S}_{7} \mathrm{C}_{8}$, WSL22 had similar bark colour (light greenish and smooth), but clones W109, FRIAM 100, FRIAM 118, WSL 22 had less smooth bark and others clones had bark colour (dark greenish and smooth), but clones FRIAM 72, FRIAM 81, Bahar had less smooth bark after six months. Several workers had also studied the disease resistance of Populus deltoides clones under 
nursery conditions. In a study five clones (D121, G-48, D-153, G-3 and 63/51) were found to be moderately resistant to five major foliage pathogens at nursery stage (Singh et al., 1991). Chandra and Joshi (1994) also reported that twenty-six clones were immune (including G-48, ST-29, ST-61, $\mathrm{S}_{7} \mathrm{C}_{1}, \mathrm{~S}_{7} \mathrm{C}_{4}$, $\mathrm{S}_{7} \mathrm{C}_{8}$, etc.) and five clones as resistant ones.

\section{Leaf parameters}

Out of 19 poplar clones, W39, W32, FRIAM 70, G48, Udai, Bahar and FRIAM100 was produced maximum number of leaves. The leaves number was ranged from 120-200. In case of Leaf Area Index was recorded highest in Bahar (378) followed by WSL 22, FRIAM 72 and W110.

The information on different leaf parameters provides an overview of morphological differences among the different clones as well as the growth pattern. In Table 6 the various clones are significantly varied among each other for different characteristics. The study indicated that there is no any relationship among number of leaves and leaf area index.

\section{Correlation study}

In the present investigation positive correlation (Table 7) was observed among various characters viz., total height, collar diameter, internodal length, number of branches, number of roots, root length and volume index at both time data recorded (T1, after six months and $\mathrm{T} 2$, after one year). It was observed that significant positive correlation was found among all the characters.

The correlation coefficient was observed higher than 0.7 among total height, collar diameter, root length with total height, collar diameter and volume index with total height, collar diameter, root length at both time of data recorded. The correlation coefficient was significantly higher in number of branches with inter-nodal length, root length with total height, collar diameter, inter-nodal length, number of roots and volume index with root length after six month as compared to one year. These results indicated the feasibility of selection of clones for higher values of total height, collar diameter, root length and volume index. The results obtained are discussed with the finding of other scientific workers. These findings confirm the reports by earlier scientific workers in different tree species. In earlier reports significant and positive correlations were observed between total height, basal diameter, number of branches, inter-nodal length, survival percentage and sprouting percentage in Populus deltoides (Verma and Bangarwa, 2005), total height and diameter in Populus ciliate (Khosla et al., 1980). Tewari et al., (1994) also reported intercharacter correlation coefficents for different growth characters in Populus deltoides. Rawat et al., (2001) also studied the inter relationship between different growth characters in Populus deltoides and reported positive correlations among all the characters viz., plant height, collar diameter, survival percentage and internodal length.

\section{References}

Alpana and Biswas, S. (1999). Taxonomic evaluation of some important clones of poplar through studies on their winter buds. Indian Journal of Forestry. 22(4): 391-393.

Chandra, J.P. and Joshi B.C. (1994). Performance of exotic clones in Tarai (U.P.). Indian Forester. 120 (2): 110-118.

Chaudhary, L. and Tewari, S.K. (2006). Identification of poplar clones through Morphological Markers of winter buds. Indian Journal of Forestry. 29(2): 135-138.

Chauhan, S.K., Sharma, S.C., Beri, V., Ritu, Yadav, S and Gupta, N. (2010). Accounting poplar and wheat productivity for carbon sequestration agri-silvicultural system. Indian Forester. 136: 1174-1182. 
Chauhan, V.K. (2008). Nursery performance of selected clones of poplar (Populus deltoides Marsh.). Indian Journal of Forestry. 31(3): 395-397.

Dhiman, R.C. (2009). Carbon footprint of planted poplar in India. Envis. Forestry Bulletin. 9: $70-81$.

Gera, M. (2012). Poplar culture for speedy carbon sequestration in India: a case study from Tarai region of Uttrakhand. Envis. Forestry Bulletin. 12: 75-83.

ICFRE. (2012). Country reports on poplars and willows. Dehradun, India: Indian counsil of Forestry Research and Education.

Karnatka, D.C, and Chandra, A. (1995). Growth observation of six poplar clones. Indian Forester. 121(1-6): 309-311.

Khosla, P.K., Kaushal, P.C. and Khurana, D.K. (1980). Studies in Populus ciliata Wall. Ex Royle. II. Phenotypic variation in natural stands. Silvae Genetica. 29: 27-31.

Kishwan, J. and Kumar D. 2013. Future of poplars in India. Rome, Italy: Food and Agricultural Organisation of the United Nations. Available online at http:/ www. Fao.org/Forestry/ 6285.

Kumar, D., Singh, N.B., Rawat, G.S., Srivastava, S. and Mohan, D. (1999). Improvement of Populus deltoides Bartr.ex Marsh. in India - I. Present status. Ind. For. 125: 245-263.

Panse, V.G. and Sukhatme, P.V. (1978). Statistically methods for agricultural workers, $3^{\text {rd }}$ ed. ICAR, New Delhi.

Rawat, G.S., Singh, N.B., Gupta, R.K., Singh, K. and Sharma S.D. (2001). Clonal evaluation of poplar (Populus deltoides Bartr.) in eastern Uttar Pradesh. I- Nursery testing. Indian Forester. 127 (1): 70-80.

Singh, N.B., Kumar, D., Rawat, G.S. and
Shrivastava, S.K. (1999). Improvement of Populus deltoides Bartr. Ex. Marsh. in India, I. Present status. Indian Forester. 125(3): 341-354.

Singh, P. and Lodhiyal, L.S. (2009). Biomass and carbon allocation in 8-year-old poplar (Populus deltoides marsh) plantation in Tarai agroforestry systems of central Himalaya, India New York Science Journal. 2(6): 49-53.

Singh, S., Pandey, P.C., Singh, A., Bahadur, R. and Mukhrerjee, S.N. (1991). Studies on foliage diseases of poplar. Indian Forester. 117(2): 135-142.

Tewari, S.K., Pandey, D., Pandey, V. and Tripathi, S. (1994). Intercharacter correlation in Populus deltoides Bartr. Indian journal of Forestry. 17(1): 61-73.

Toky, O. P., Bisht, R. P., Kumar, N., Singh, R. R. (1996). Growth variability of Populus deltoides Marsh. clones in arid climate of North-Western India. Indian J Forest. 19 (1): 69-73.

Verma, R.C. (2001). Studies on genetic variation and clonal testing in Populus deltoides Bartr. Ex. Marsh. Ph. D. Thesis submitted to CCS HAU, Hisar.

Verma, R.C. and Bangarwa, K.S. (2003). Performance of Populus deltoides clones under nursery conditions in arid climate of NW INDIA. Range Mgmt. Agroforestry. 24(2): 174-176.

Verma, R.C. and Bangarwa, K.S. (2005). Intercharacter correlation studies in Populus deltoides Bartr. Ex. Marsh. Clones under nursery conditions. Indian Journal of Forestry. 28(4): 359-362.

\section{How to cite this article:}

Rajiv Kumar, Bimlendra Kumari, K.K. Bhardwaj, Ashwani Kumar and Tarun Kumar. 2017. Study on Growth and Phenotypic Characters of Different Clones of Poplar (Populus deltoides Marsh.) in Nursery. Int.J.Curr.Microbiol.App.Sci. 6(12): 1840-1848. doi: https://doi.org/10.20546/ijcmas.2017.612.209 